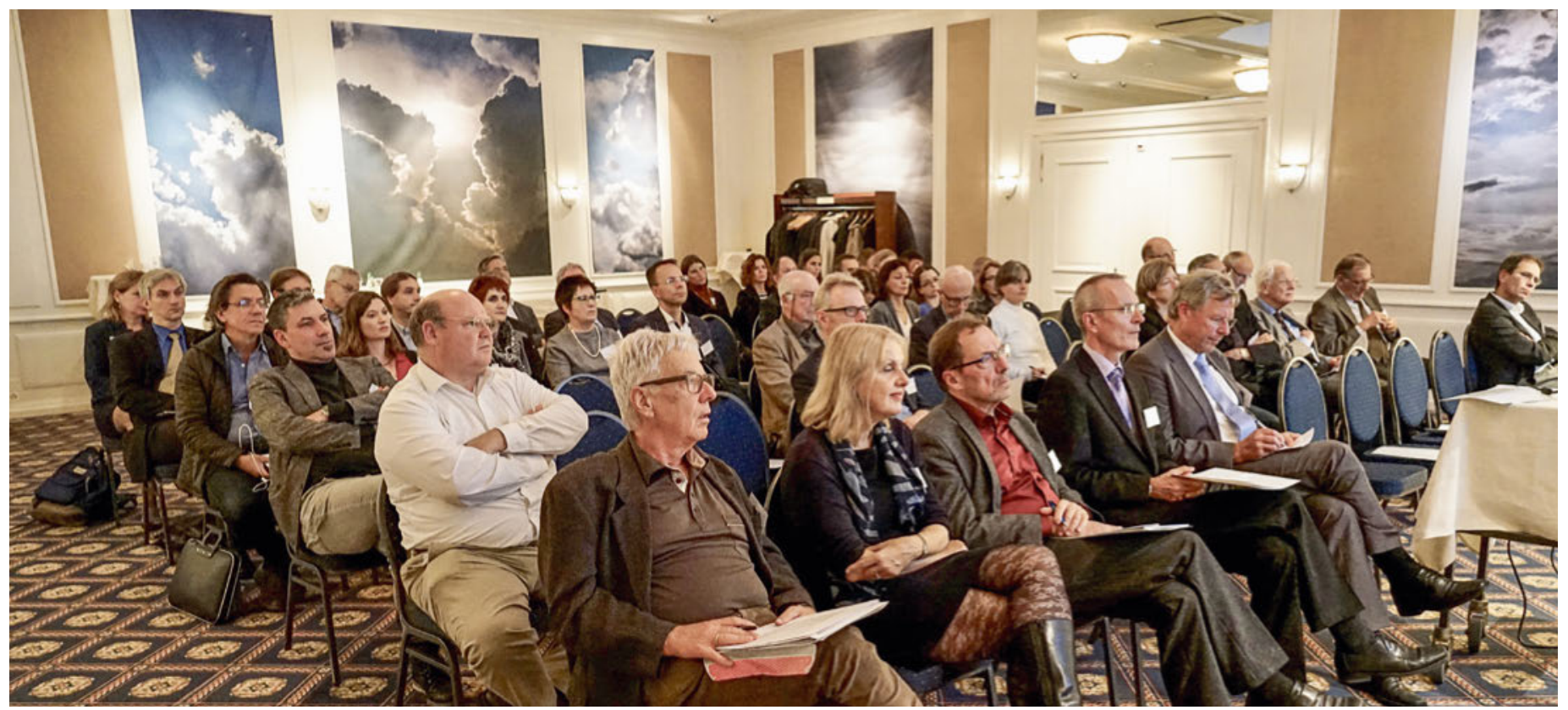

\title{
Von dunklen Wolken und blauem Himmel
}

\section{Matthias Scholer}

Redaktor Schweizerische Ärztezeitung

Welche Informationsquellen nutzen Sie für Ihre berufliche Tätigkeit? Lesen Sie regelmässig medizinischen Fachzeitschriften? Lieber Online oder Print? Antworten auf diese und viele weitere Fragen liefert eine aktuelle gsf-Umfrage. Deren Auswertung bildete neben dem 20-Jahr-Jubiläum des Schweizerischen Ärzteverlags den Kernpunkt der diesjährigen Sitzung der Redaktionskommissionen, an der zahlreiche Delegierte medizinischer Fachgesellschaften teilnahmen.

«Ab 2019 werden wir keine Abonnementsgebühr mehr für die SÄZ, SMF und SMW erhalten", gab Ruedi Bienz in seiner Eröffnungsansprache der diesjährigen Sitzung der Redaktionskommission bekannt. Ein Meilenstein und vielleicht auch ein Wendepunkt für den Schweizerischen Ärzteverlag EMH. Schliesslich sind die Schweizerische Ärztezeitung (SÄZ), das Swiss Medical Forum (SMF) und das Swiss Medical Weekly (SMW) die "Kernprodukte» des Verlagshauses, das Ruedi Bienz noch bis Ende Jahr leitet. Bienz erinnerte die Teilnehmenden aber auch an einen weiteren Mei- lenstein, der vor lauter Finanzfragen fast etwas in den Hintergrund rückte: das 20-Jahr-Jubiläum des EMH Verlags. Dieser wurde 1997 von der FMH zusammen mit dem traditionsreichen Basler Verlagshaus Schwabe ins Leben gerufen. Die Kollaboration sollte dazu dienen, einerseits die Kommunikation mit den FMHMitgliedern zu professionalisieren und andererseits den Ärztinnen und Ärzten unabhängige und qualitativ hochstehende Publikationen in den Bereichen Gesundheitspolitik, Aus-, Fort- und Weiterbildung, aber auch Wissenschaft und Forschung anzubieten. 


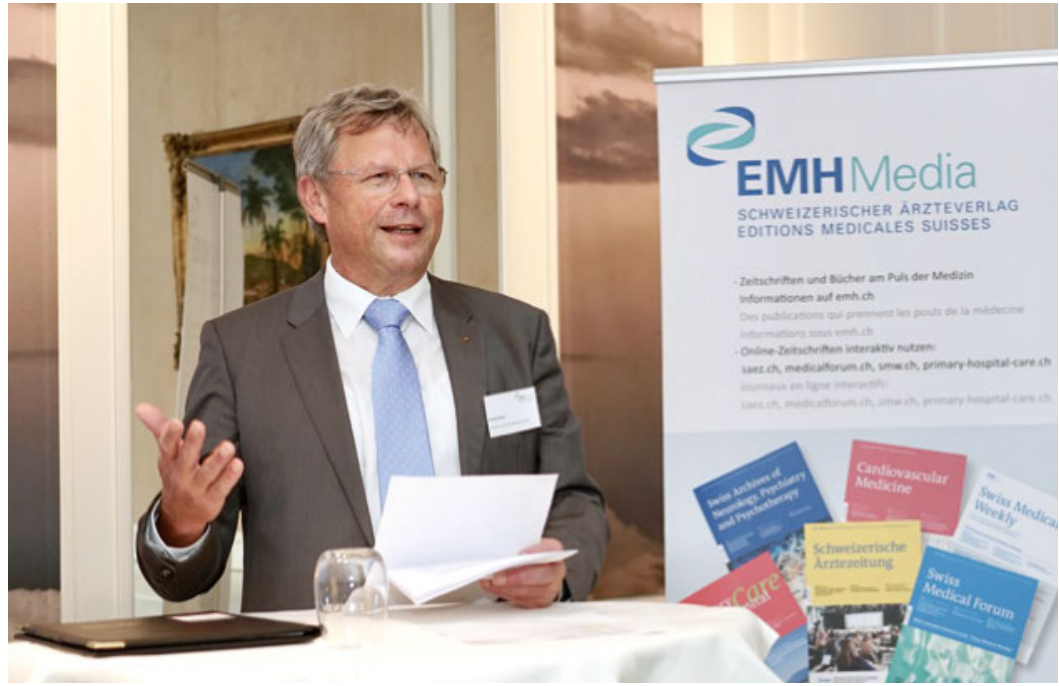

Der scheidende EMH-Geschäftsleiter Ruedi Bienz.

Wurde dieses Ziel erreicht? Eine Frage, die nur von der Leserschaft beantworten werden kann.

Da das Markt- und Sozialforschungsunternehmen gfszürich eine Wiederholung der 2010 durchgeführten Umfrage «Medizinische Fachpresse» plante, nutzen die FMH und EMH die Gelegenheit, um spezifische Fragen zu den eigenen Produkten in den aktuellen Fragekatalog einfliessen zu lassen. Andreas Schaub, Leiter gfszürich, erläuterte im Anschluss an Ruedi Bienz' Begrüssungsworte die wichtigsten Resultate und Eckwerte der Studie. ${ }^{1}$

\section{Das duale Modell als Erfolgsrezept}

An der Umfrage nahmen 2550 Ärztinnen und Ärzte aus allen Landesregionen und verschiedenster Fachrichtungen teil. Hauptziel der Studie war es, herauszufinden, welche medizinischen Fachzeitschriften von der Zielgruppe aktuell gelesen werden und wie deren Qualität eingeschätzt wird. Aus Sicht der EMH sind gleich mehrere Resultate erfreulich und vielversprechend Die EMH-Zeitschriften haben einen sehr hohen Bekanntheitsgrad, werden gut gelesen und erfreuen sich grosser Beliebtheit. Und: Die befragten Ärztinnen und Ärzte schätzen die redaktionelle Unabhängigkeit medizinischer Fachzeitschriften als wichtig bis sehr wichtig ein, wie die Abbildung 1 zeigt.

Nach der Präsentation der Umfrageergebnisse informierten die einzelnen Chefredaktoren über Neuigkeiten rund um die von ihnen betreuten Zeitschriften. Bruno Kesseli nutzte dabei die Gelegenheit, den anwesenden Gästen das duale Modell der SÄZ in Erinnerung zu rufen. So übernimmt die Schweizerische Ärztezeitung einerseits die Funktion eines "Amtsblatts", wie Kesseli augenzwinkernd den vorderen Teil der Zeitschrift nannte, welcher der FMH als offizielles Organ dient. Der hintere Teil der SÄZ hingegen steht der Ärzteschaft und den Fachgesellschaften als redaktionell betreute Informations- und Diskussionsplattform zur Verfügung.

Dass die Leserschaft mit diesem Inhaltskonzept zufrieden ist, spiegelt sich in den Umfrageergebnissen wider.

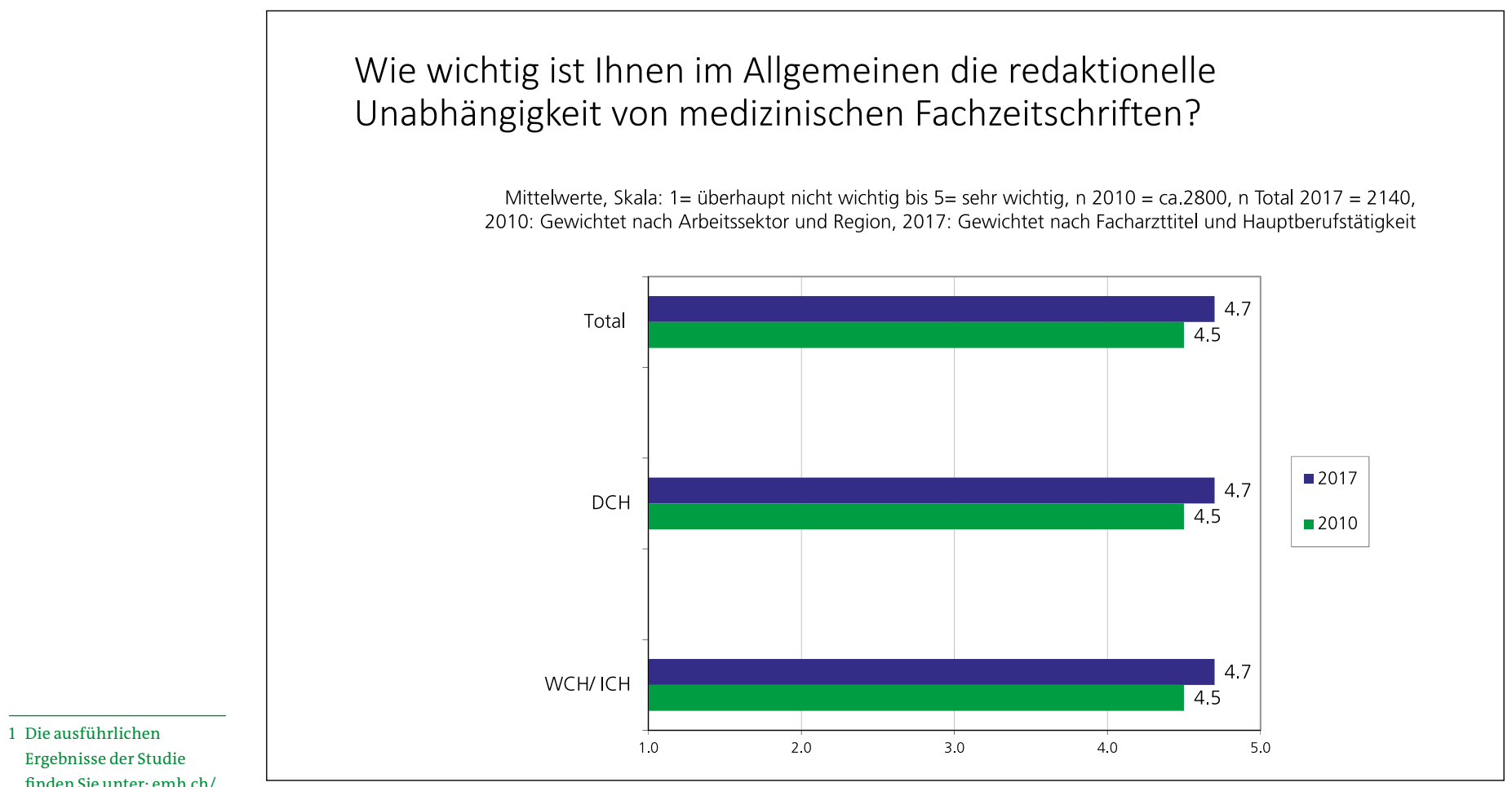


Schweizerische Ärztezeitung

Wie lesen Sie die einzelnen Rubriken?

Alle Ärzte

Angaben in Prozent, Skala: nie bis immer, n Total $2017=2124$, Gewichtet nach Facharzttitel und Hauptberufstätigkeit, Filter: falls SÄZ in den letzten sechs Monaten mindestens einmal in der Hand gehabt, um darin zu blättern oder zu lesen (Frage WLK)

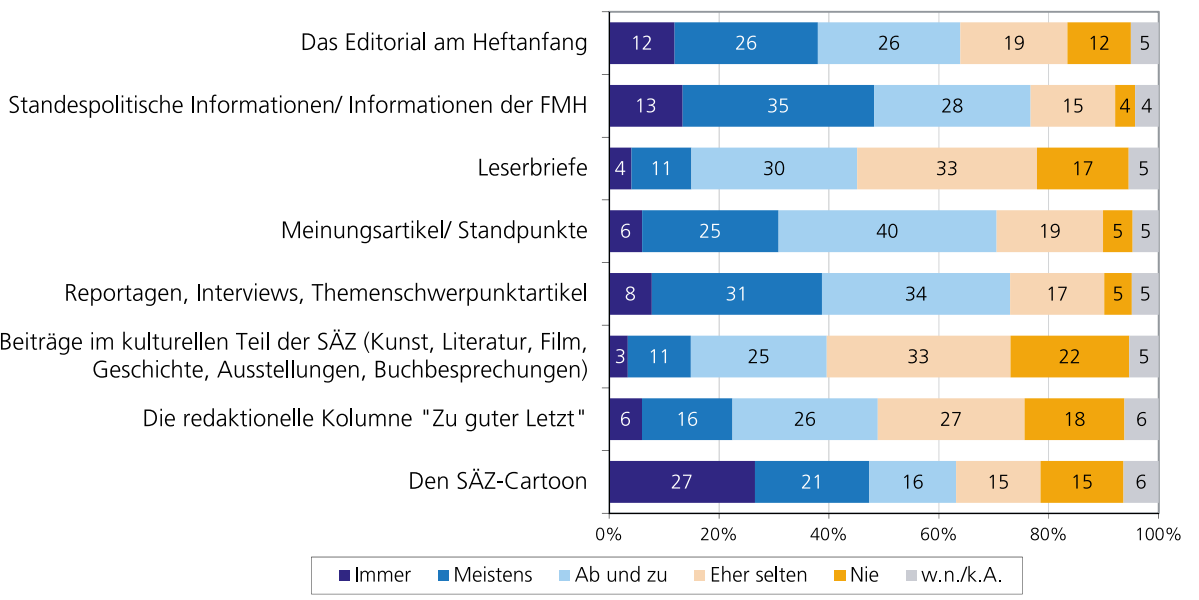

Abbildung 2: Das Inhaltskonzept der SÄZ kommt grundsätzlich gut an.

So gefällt der Mehrheit der Befragten der thematische Mix der Beiträge, wie auch die Tatsache, dass die Zeitschrift den Ärztinnen und Ärzten und weiteren Kreisen im Gesundheitswesen offensteht. Welche Rubriken bei der Leserschaft am meisten Anklang finden, zeigt die Abbildung 2.

Im letzten Jahr sei das Informationsangebot der SÄZ mit zeitgemässen Online-Features ergänzt worden, betonte Kesseli. So können unter der neu geschaffenen Website-Rubrik «Tour d'horizon» Themen aus dem Gesundheitswesen schnell und multimedial aufgegriffen werden. Ebenfalls als ergänzende Dienstleistung ist der neue Facebook-Auftritt der SÄZ zu sehen, der bislang erst in deutscher Sprache angeboten wird. Die Aufschaltung des französischen Social-Media-Kanals ist auf Anfang nächsten Jahres geplant.

\section{Grosse Herausforderungen warten auf den EMH}

Hans Kurt, VR-Präsident des EMH Verlags, fasste zum Abschluss des Anlasses das Gehörte zusammen. Dabei nutzte er die Wolkenmalerei auf den Festsaalwänden als Metapher für die momentane Situation: Einerseits dunkle Gewitterwolken, welche für die grossen Herausforderungen stehen, mit denen sich der EMH Verlag nach dem Wegfall der Abonnementsgebühren konfrontiert sieht. Kann unter diesen Bedingungen die redaktionelle Unabhängigkeit und die hohe Qualität der EMH-Zeitschriften aufrechterhalten werden? Doch die Wandmalerei zeigt zwischen den Wolken auch blauen Himmel - für Hans Kurt das Symbol der Zuversicht, dass die EMH-Produkte auch künftig den Schweizer Ärztinnen und Ärzten als wichtige Informationsquelle in ihrem Berufsalltag dienen werden. Schliesslich stehen hinter den einzelnen Fachmedien engagierte Redaktionsmitglieder und professionelle Verlagsmitarbeiter, die eine SÄZ, ein SMF und ein SMW erst zu dem machen, was sie sind.

\section{Bildnachweis}

Grafiken: gfs-zürich

Fotos: Matthias Scholer 